\title{
Effects of Cross-Linking Density and of Stretching on Dielectric $\alpha$-Relaxation in Poly(acrylonitrile-co-butadiene) Rubber
}

\author{
Hiroshi ADACHI, Keiichiro ADACHI, and Tadao KOTAKA* \\ Department of Polymer Science, Osaka University, \\ Machikaneyama, Toyonaka, Osaka 560, Japan.
}

(Received December 12, 1979)

\begin{abstract}
Dielectric studies were performed on acrylonitrile-butadiene random copolymer with intermolecular carbon-carbon cross-links by dicumyl peroxide. The effects of cross-linking and of stretching on the $\alpha$-relaxation were examined on the polymer coated with evaporated-gold electrodes. With increasing cross-linking density the strength of dielectric relaxation decreased, the position of loss maximum shifted to a higher temperature, and the half width of the absorption curve increased. The apparent activation energy was virtually unchanged. On the other hand, the effect of stretching on the $\alpha$-relaxation could not be observed in the range of the elongation ratio upto 3 examined in these experiments. These results may be interpreted as due to the decrease in free volume, especially in those regions close to the cross-links and hence, to the broadening of the distribution of free volume.
\end{abstract}

KEY WORDS Acrylonitirle-Butadiene Rubber / Dielectric $\alpha$-Relaxation /

Effect of Cross-Linking Density / Effect of Stretching /

A large number of studies have been carried out on the viscoelastic properties of cross-linked rubbery polymers. ${ }^{1-3}$ However, studies on the dielectric relaxation are rather few. ${ }^{2,3}$ Bakule and Havranek ${ }^{4}$ studied the dielectric properties of natural rubber, synthetic polyisoprene, and cis-polybutadiene vulcanized with sulfur, examining the effects of crosslinking density on the dielectric $\alpha$-relaxation associated with the micro-Brownian motion of network chains. They observed, with increasing sulfur content, (i) an increase in the relaxation strength $\Delta \varepsilon$, which is the difference in the dielectric constant $\varepsilon^{\prime}$ measured at low and high frequencies, (ii) a shift of the dielectric loss maximum temperature $T_{\max }$ to a higher temperature at a given frequency, and (iii) an increase in the half width $\Delta h$ of the dielectric absorption curves $\varepsilon^{\prime \prime}(\omega)$. They ascribed these changes to the increasing restraint on the segmental motions with an increasing degree in cross-linking. In the sulfur vulcanization of polydienes, it is known that side-reactions resulting in polar heterocyclic groups take place. ${ }^{3,5}$ Therefore their observations might have been obscured by the motions of such groups built into the polydiene chains. On the other hand, Nose et al. ${ }^{6,7}$ studied the effects of chain

\footnotetext{
* To whom correspondence should be addressed.
}

stretching on the dielectric $\alpha$-relaxation in partly chlorinated polyethylene and polychloroprene, using $\mathrm{Hg}-\mathrm{Th}$ alloys as the electrodes. They reported that (i) $T_{\max }$ shifts to higher temperature, (ii) $\Delta \varepsilon$ decreases and (iii) $\Delta h$ increases, as the test specimen being stretched upto the extension ratio $\lambda=2$. In order to avoid the electrode effects, ${ }^{2}$ usually vacuumevaporated metal electrodes are used, deposited on the clean surfaces of the specimen. However, it is nearly impossible to achieve a satisfactory coating on a rubbery polymer with an evaporated metal layer, especially when the coating is carried out above the glass-transition temperature, $T_{\mathrm{g}}$, of the specimen.

Thus, we have attempted to draw a more definite conclusion on the effects of cross-linking density and of the stretching on the dielectric $\alpha$-relaxation in polymer networks, avoiding these difficulties by improving the experimental procedures. To this end, we employed an acrylonitrile-butadiene randomcopolymer rubber (NBR) and vulcanized it to various extent with dicumyl peroxide. The reagent, unlike sulfur, introduces only carbon-carbon crosslinks but no polar groups into the network. ${ }^{3,8}$ To achieve a satisfactory coating on NBR with an evaporated gold layer, we devised an evaporator which can deposit gold onto a surface of an NBR specimen while it is being cooled below the $T_{\mathrm{g}}$ 
through the other surface and stretched upto $\lambda=3$. Herein these results are reported.

\section{EXPERIMENTAL}

\section{Materials}

An emulsion-polymerized NBR sample with an acrylonitrile content of $29 \%$ by weight was provided at the courtesy of Dr. Tagata of Japan Synthetic Rubber Co. The sample was cross-linked with $2 \mathrm{wt} \%$ dicumyl peroxide at $120^{\circ} \mathrm{C}$ with varying vulcanization time. ${ }^{8}$ Impurities remaining in the crosslinked specimens were removed by hot-solvent extraction for $4 \mathrm{~h}$ with the azeotropic mixture of ethanol, benzene, and water with the volume ratio $7: 3: 1$, respectively. ${ }^{10}$ The specimens were thoroughly dried in vacuum at about $50^{\circ} \mathrm{C}$ for 8 to 10 days. The weight loss after this treatment was about $5 \%$. An acrylonitrile group has a dipole moment of about 4 debye units, ${ }^{9}$ and is fixed perpendicular to the main chain. Therefore, the main contribution to the dielectric $\alpha$-relaxation should come from the motion of the acrylonitrile groups induced by the main-chain segmental motions, and those of the cross-links and other impurities should be neglected. Table I summarizes the condititions of cross-linking and the characteristics of the specimens as determined by Young's moduli measurements on a Rheovibron DDV-II (Toyo Baldwin Co.). The density of effective cross-links $v$ was defined from the Young's modulus $E$ as $v=E / 3 R T$, where $R T$ has the usual meaning.

\section{Apparatus and Dielectric Measurements}

For dielectric measurements a mutual inductance bridge (Showa Denki Laboratories, Tokyo) was employed to cover the frequency range $3.125 \mathrm{~Hz}$ to $1.6348 \mathrm{MHz}$. To carry out reliable measurements, the use of vacuum-evaporated metal electrodes was essential. However, the coating of a rubbery specimen is usually tedious and the metal does not form perfectly conducting layers on the rubbery specimen. To avoid this difficulty we devised a low-temperature vacuum evaporator ${ }^{11}$ such as shown in Figure 1. With this device, we coated both surfaces of the cross-linked NBR specimens with gold, usually, $3 \mathrm{~cm}$ in diameter and $1 \mathrm{~mm}$ in thickness.

The dielectric relaxation strength $\Delta \varepsilon$ of a goldcoated specimen was about $5 \%$ more than that of a

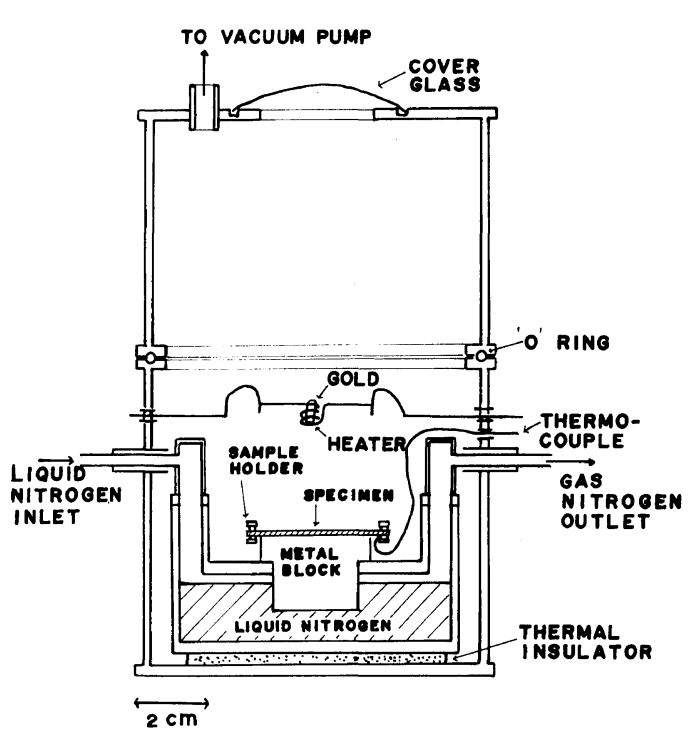

Figure 1. A low-temperature vacuum evaporator for coating polymers having low glass-transition temperatures.

Table I. Characteristics of cross-linked NBR specimens ${ }^{\mathrm{a}}$

\begin{tabular}{|c|c|c|c|}
\hline \multirow{2}{*}{$\begin{array}{c}\text { Code } \\
\text { No. }\end{array}$} & \multirow{2}{*}{$\frac{\text { Curing time }}{\min }$} & \multirow{2}{*}{$\frac{\text { Young's modulus, } E \times 10^{-6}}{\mathrm{~N} \mathrm{~m}^{-2}}$} & \multirow{2}{*}{$\frac{\text { Cross-linking density, } \times 10^{4}}{\mathrm{~mol} \mathrm{~cm}^{-3}}$} \\
\hline & & & \\
\hline 1 & 5 & 1.0 & 1.4 \\
\hline 2 & 10 & 1.3 & 1.8 \\
\hline 3 & 20 & $2.0^{\mathrm{b}}$ & 2.8 \\
\hline 4 & 40 & 2.4 & 3.3 \\
\hline 5 & 80 & 3.1 & 4.2 \\
\hline
\end{tabular}

a With $2 \mathrm{~g}$ peroxide $/ 100 \mathrm{~g} \mathrm{NBR}$ cured at $120^{\circ} \mathrm{C}$.

b Measured at $289 \mathrm{~K}$, otherwise at $294 \mathrm{~K}$. 
corresponding uncoated specimen simply sandwiched between metal-block electrodes. The difference of $5 \%$ is about twice as much as the experimental error encountered in an ordinary experiment. The difference became much larger, when the specimen was stretched, as was already pointed out by Gemant. ${ }^{12}$ In our dielectric measurements ${ }^{11}$ a goldcoated specimen was mounted on a three-electrode cell. The cell was then placed in an air-tight brass container. The container was evacuated for several hours and then filled with dry helium gas to prevent moisture.

For measurements on a stretched specimen, a rubber sample was stretched at room temperature up to a given ratio, held between a cramp, and then coated with gold in the vacuum-evaporator. It took about $10 \mathrm{~h}$ to complete the coating on both surfaces. In this way we carried out the dielectric measurements under the condition that the electric field was applied perpendicular to the direction of elongation.

During the frequency dispersion measurements, the temperature was regulated to within $0.1 \mathrm{~K}$ around the desired value with a thermobox (Shinnihon Denko Ltd., Tokyo). For the temperature dispersion measurements, the heating rate of $0.3 \mathrm{~K}$ $\min ^{-1}$ was maintained.

\section{RESULTS AND DISCUSSION}

Figure 2 shows the frequency dependence of $\varepsilon^{\prime}$ and $\varepsilon^{\prime \prime}$ of the sample No. 1 at three different temperatures. Only one relaxation is observed. The reduced absorption curve $\varepsilon^{\prime \prime}(\omega) / \varepsilon^{\prime \prime}{ }_{\text {max }}$ becomes narrower with increasing temperature, as is usually the case. Figures 3 to 5 , respectively, show the temperarure dependence of $\varepsilon^{\prime}$ and $\varepsilon^{\prime \prime}$, the reduced absorption curves, and the Cole-Cole plots for the NBR specimens with varying $v$. These figures clearly indicate that (i) the relaxation strength decreases, (ii) the halfwidth $\Delta h$ of the absorption curve becomes wider, and (iii) the loss maximum temperature $T_{\max }$ shifts to higher temperatures, as the cross-linking density $v$ increases. However (iv) the apparent activation energy $\Delta H$ shows no change at all with increasing $v$, as shown in Figure 6, which compares the activation plots, i.e., the plots of $\log$ (frequency $f$ ) vs. $T_{\max }{ }^{-1}$ for the NBR specimens.

Effects of stretching on the temperature and the frequency dependence of $\varepsilon^{\prime}$ and $\varepsilon^{\prime \prime}$ were examined on the most lightly cross-linked specimen No. 1 with the

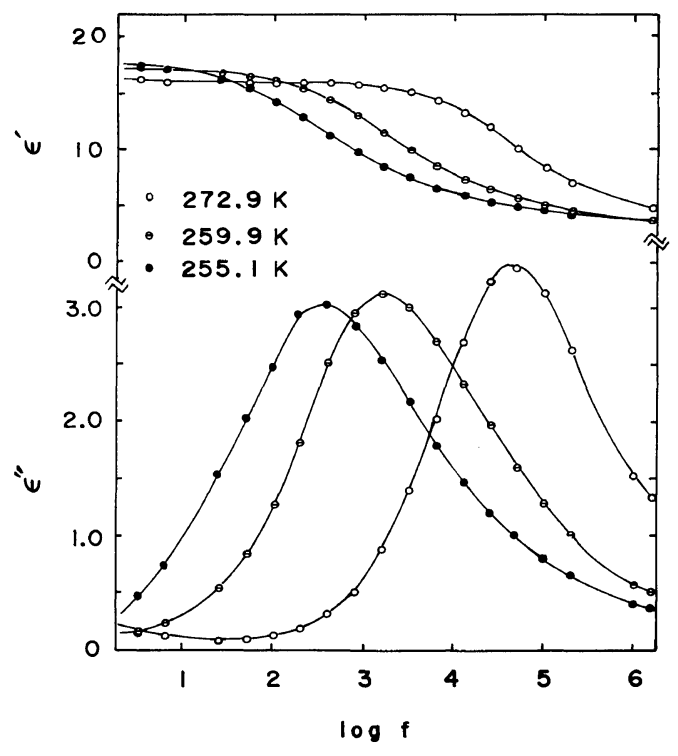

Figure 2. Frequency dependence of $\varepsilon^{\prime}$ and $\varepsilon^{\prime \prime}$ at various fixed temperatures for the NBR gold-coated specimen No. $1\left(v=1.4 \times 10^{-4} \mathrm{~mol} \mathrm{~cm}^{-3}\right)$.

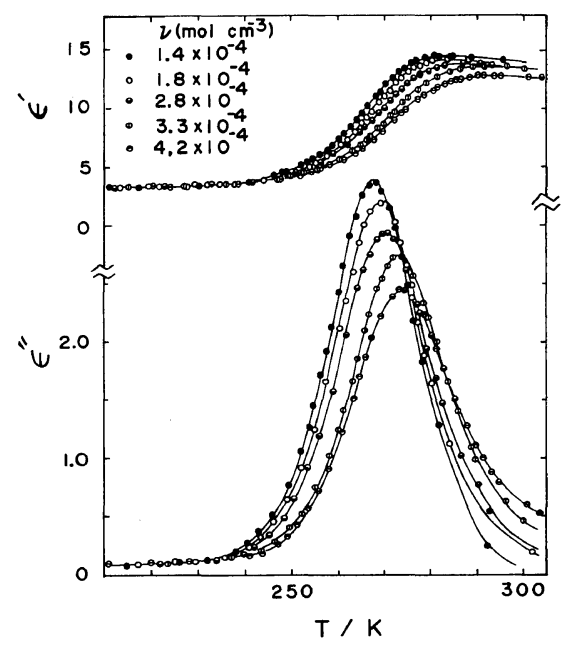

Figure 3. Temperature dependence of $\varepsilon^{\prime}$ and $\varepsilon^{\prime \prime}$ at 12.8 $\mathrm{kHz}$ for the NBR gold-coated specimens with various cross-linking densities $(v)$ as indicated.

evaporated-gold coating. The results are shown in Figures $7 \mathrm{a}$ and $7 \mathrm{~b}$. For the sake of comparison, Figure 8 shows the effects of strecthing on the frequency dependence of $\varepsilon^{\prime}$ and $\varepsilon^{\prime \prime}$ for the same sample but without the coating. The specimen 


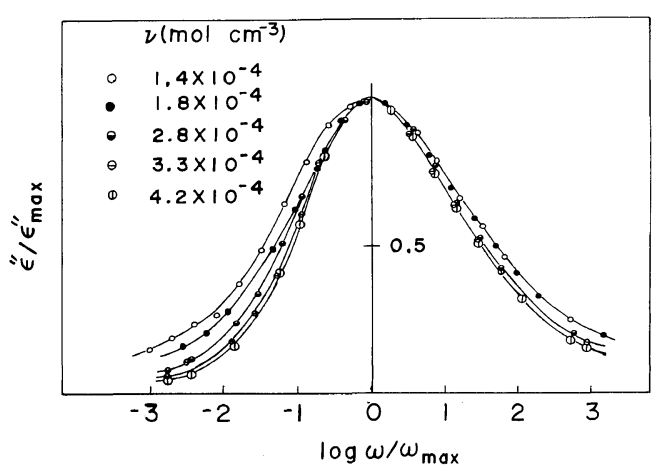

Figure 4. $\varepsilon^{\prime \prime} / \varepsilon^{\prime \prime}{ }_{\max }$ for the NBR gold-coated specimens with various $v$ as indicated.

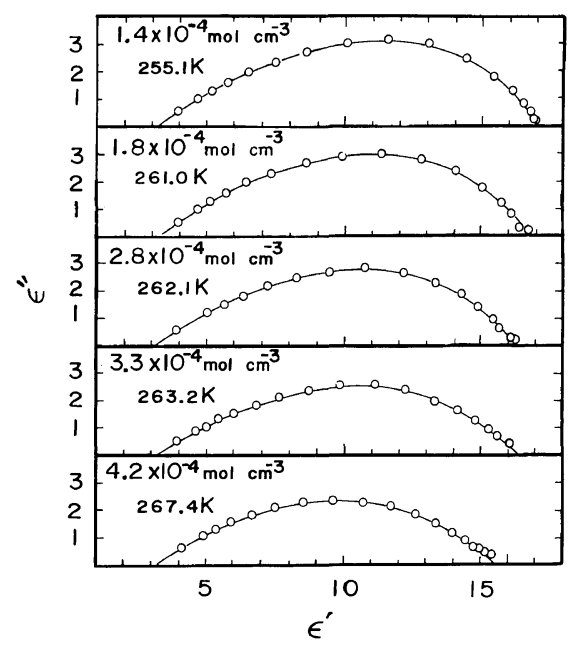

Figure 5. Cole-Cole plots for the NBR gold-coated specimens with various $v$ as indicated.

without the coating exhibits a substantial decrease in $\Delta \varepsilon$ by stretching, while the specimen with the coating exhibits no change at all. Similar results were observed in other specimens with a higher degree of cross-linking.

In the dielectric relaxations, the frequency of the absorption maximum $f_{\max }$, the half-width $\Delta h$, and the dielectric relaxation strength $\Delta \varepsilon$ are related, respectively, to the mean relaxation time, the distribution of the relaxation times, and the number of dipoles (multiplied by the effective moment) involved in the particular relaxation process, ${ }^{2,3}$ which is, in this case, the $\alpha$-relaxation due to the main chain motions. The effects of various factors on the $\alpha$ -

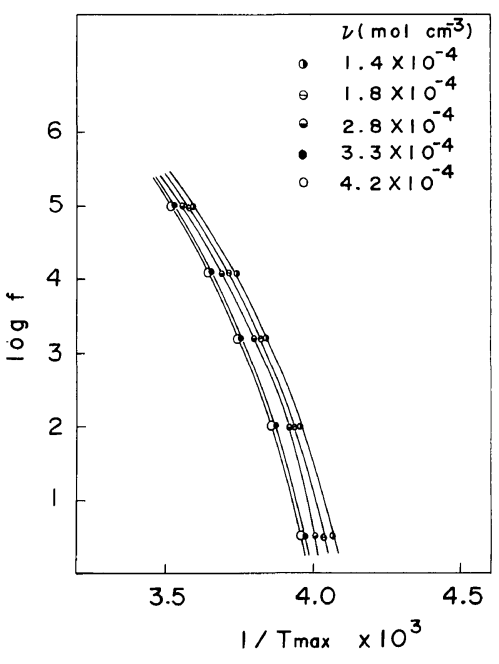

Figure 6. Activation plots for the NBR gold-coated specimens with various $v$ as indicated.

relaxation have been extensively studied so far, including such factors as isotropic external pressure, ${ }^{13}$ degree of crystallinity, ${ }^{14-16}$ and main-chain alignment due to cold drawing. ${ }^{17}$ The general conclusions drawn from such studies ${ }^{2.3}$ are that (i) a shift of the loss maximum position, i.e., $f_{\max }$ at a fixed temperature to lower frequency or $T_{\max }$ to higher temperature, (ii) a broadening of $\Delta h$, and (iii) a decrease in $\Delta \varepsilon$ will be caused by any of such factors which might reduce the segmental mobilities or set the restraint on the segmental motions. These effects may be brought about by an decrease in free volume, an increase in the interactions between partially aligned chain segments ${ }^{17}$ (interchain effects), and/or the change in stereochemical conformations toward more unstable and restrictive ones, leading to the change in transitional rates among the available rotational isomeric states ${ }^{18}$ (an intrachain effect).

As to the mechanical $\alpha$-relaxation in cross-linked rubbers with dicumy peroxide, Mason ${ }^{19}$ observed that an increased degree of cross-linking makes the specific volume decreased, $T_{\mathrm{g}}$ raised and the transition region broadened. Mason interpreted these observations as the result of restricted microBrownian motion of network chains due to the decrease as well as broadening of the distribution of available free volumes. ${ }^{19}$ The present results on the dielectric $\alpha$-relaxation in the cross-linked NBRs may be summarized as in Figure 9, where $T_{\max }, \Delta h$, and $T \Delta \varepsilon$ are plotted against the density of effective cross- 


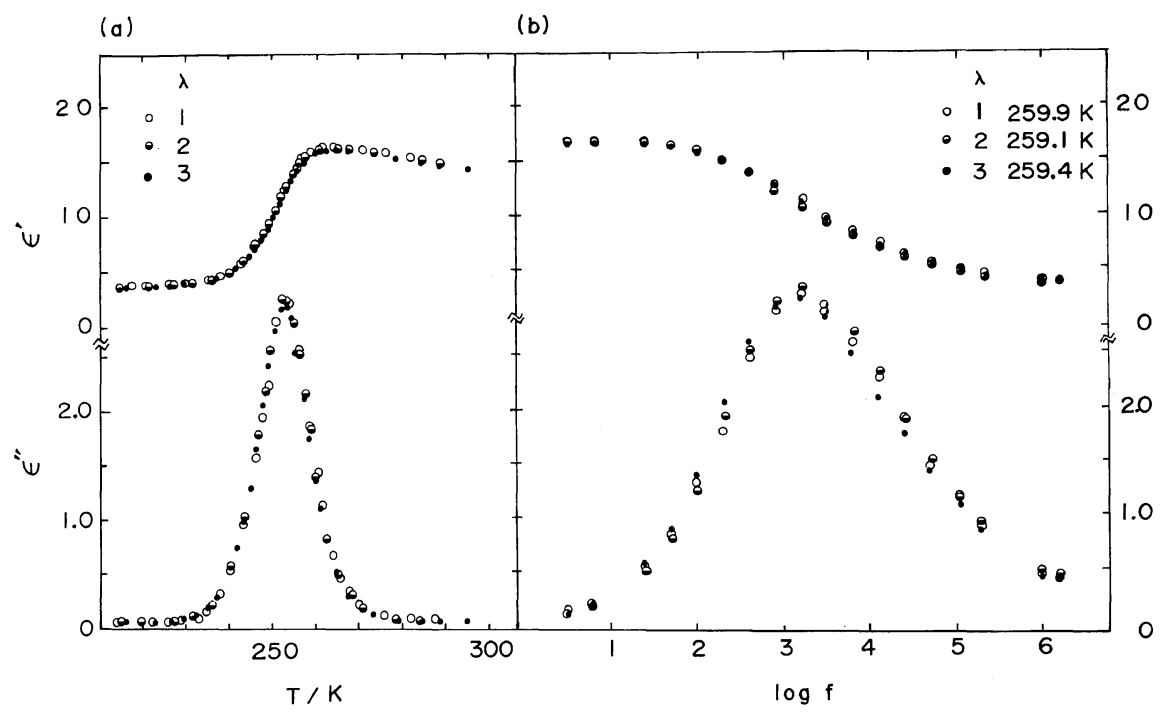

Figure 7. Effects of stretching on (a) the temperature and (b) the frequency dependences of $\varepsilon^{\prime}$ and $\varepsilon^{\prime \prime}$ for the NBR specimens No. 1 with the evaporated-gold coating.

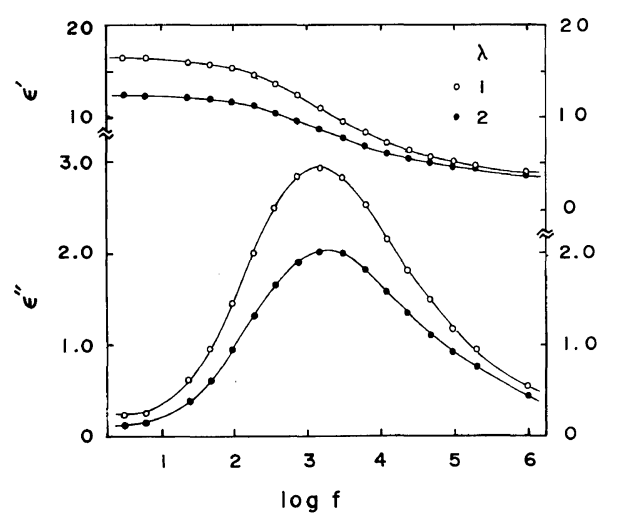

Figure 8. Frequency dependence of $\varepsilon^{\prime}$ and $\varepsilon^{\prime \prime}$ (at 259K) under various extension ratio $\lambda$ for the NBR specimen No. 1 without the coating.

links $v$. The behavior of the former two quantities are in accord with those of the mechanical $\alpha$ relaxation. The last quantity $T \Delta \varepsilon$, which was not dealt with in Mason's work, ${ }^{19}$ is found to decrease approximately linearly with $v$. This feature appears to reflect a decrease in the number of actual motional dipoles involved in the $\alpha$-relaxation. Such an decrease is due more likely to the decrease in available free volume as a result of increased cross-linking density rather than to the restraint on the large-scale segmental motions of the network chains (the in-

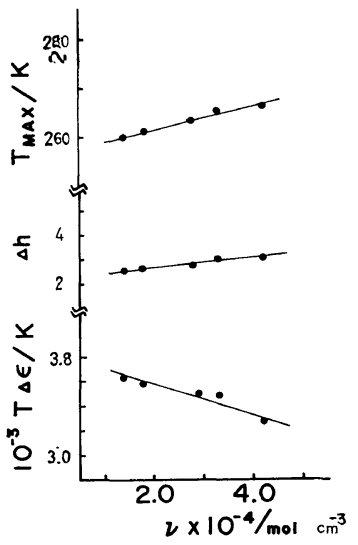

Figure 9. Dependence of the position of loss maximum $T_{\max }(1.6 \mathrm{kHz})$, the half-width $\Delta h$ of the absorption curve, and the strength of relaxation $T \Delta \varepsilon$ on the cross-linking density $v$.

trachain effect).

In connection with these observations, the effects of stretching are noteworthy in the sense that we did not see any effects on the permittivity and loss, at a direction perpendicular to that of stretching. Infrared dichroism measured on the NBR specimen No. 1 stretched at $\lambda=3$ indicated that about $20 \%$ orientation has taken place along the direction of stretching. Since the polar groups in NBR attached perpendicular to the main chain, we expected an 
increase in the relaxation strengh, as Peterlin and Elwell had pointed out. ${ }^{17}$ However, it turned out that the stretching resulted in no effects on the goldcoated specimens. The effects seen in the uncoated specimen were mere artifacts due presumably to poor contact between the polymer and electrode surfaces. This observation may imply that the elongation of network chains might proceed in non-affine manners. Cross-links do not exert any significant influence on the segmental motions remote from the cross-links but only on those close to them through the reduction of local free volumes near the crosslinks, ${ }^{19}$ thereby leading to a decrease in the overall free-volume fraction and to the broadened inhomogeneous distribution. Such an idea might apply to the effects of increasing degree of crystallinity in the dielectric and/or mechanical $\alpha_{\mathrm{a}}$-relaxations of semicrystalline polymers.

\section{REFERENCES}

1. See, for example, J. D. Ferry, "Viscoelastic Properties of Polymers," 2nd ed, John Wiley, New York, N.Y., 1970, p 431.

2. See also, N. G. McCrum, R. E. Read, and G. Williams, "Anelastic and Dielectric Effects in Polymer Solids,” John Wiley, London, 1967.
3. See also, P. Hedvig, "Dielectric Spectroscopy of Polymers," Akademiai Kiado, Budapest, 1977, p 312.

4. R. Bakule and A. Havranek, J. Polym. Sci., C, 53, 347 (1975).

5. E. H. Farmer and F. W. Shipley, J. Chem. Soc., 2, 1519 (1947).

6. M. Naoki, K. Nakajima, T. Nose, and T. Hata, Polym. J., 6, 283 (1974).

7. K. Nakajima, M. Naoki, and T. Nose, Polym. J., 10, 307 (1978).

8. F. R. Eirich, Ed., "Science and Technology of Rubber," Academic Press, New York, N.Y., 1978, p 291.

9. V. I. Minkin, O. A. Osipov, and Yu. A. Zhdanov, "Dipole Moments in Organic Chemistry," Plenum Press, New York, N.Y., 1970, p 91.

10. M. Kolthoff, C. W. Can, and B. J. Cari, J. Polym. Sci., 2, 637 (1947).

11. H. Adachi, MS Dissertation, Department of Polymer Science, Osaka University, 1977.

12. A. Gemant, Z. Phys., 73, 526 (1932).

13. G. Williams, Trans. Faraday Soc., 60, 1556 (1964).

14. Y. Ishida, K. Yamafuji, H. Ito, and M. Takayanagi, Kolloid Z. Z. Polym., 184, 97 (1962).

15. Y. Ishida, J. Polym. Sci., A-2, 7, 1835 (1969).

16. H. Adachi, K. Adachi, Y. Ishida, and T. Kotaka, $J$. Polym. Sci., Polym. Phys. Ed., 17, 851 (1979).

17. A. Peterlin and J. Elwell, J. Mater. Sci., 2, 1 (1967).

18. K. Adachi, H. Adachi, and Y. Ishida, Rep. Prog. Polym. Phys. Jpn., 18, 419 (1975).

19. P. Mason, Polymer, 5, 625 (1964). 